\title{
L'image de la maison qui brûle : figures du temps dans quelques romans d'expression française du Canada
}

\author{
Jean Morency \\ Université de Moncton
}

Je le vois comme si c'était maintenant, Quand ils venaient brûler ma maison; Chantant leurs vilaines chansons, Tenant leurs torches dans leurs vilaines mains; Dans le Nord Canadien. (Zachary Richard, «Dans le Nord Canadien »)

Le rapport au temps dans les romans d'expression française du Canada s'exprime dans un contexte particulier, caractérisé par une relation souvent difficile avec l'altérité : comment en effet « devenir Soi avec les autres » quand justement l'Autre semble 
vous dénier le droit d'exister dans l'espace et dans le temps? Inscrits dans un vaste territoire et issus de communautés fragiles, souvent privées de traces du passé et de lieux de mémoire, les romans franco-canadiens prennent forme dans un milieu où les figures du temps sont suspectes, tenues à distance, pour ne pas dire malmenées, non seulement par l'Autre majoritaire, mais aussi par le Moi minoritaire. Symptomatiques d'une américanité qui s'impose souvent avec brutalité et qui ne correspond pas à un choix librement consenti par les individus, les romans acadiens, franco-ontariens et franco-manitobains entretiennent ainsi avec les figures du temps une relation problématique et paradoxale, partagés qu'ils sont entre la nécessité de préserver une certaine mémoire liée à l'identité, d'une part, et la méfiance ressentie à l'égard de l'Histoire officielle, méfiance qui se conjugue à la tentation de s'affranchir du passé collectif, d'autre part. Ceci s'avère particulièrement vrai dans le contexte de la postmodernité, marqué par l'individualisme et la subjectivité, ainsi que par le rejet des grands récits collectifs. Dans le texte qui suit, je me propose d'étudier cette relation avec le temps (ainsi qu'avec l'altérité) en m'appuyant sur une image qu'on peut qualifier de récurrente, celle de la maison qui brûle, image présente dans l'extrait de Zachary Richard cité en exergue et dans certains romans publiés à la fin du $\mathrm{XX}^{\mathrm{e}}$ siècle, principalement dans Histoire de la maison qui brûle(1985) de France Daigle et L'Obomsawin (1987) de Daniel Poliquin, mais aussi dans Un vent se lève qui éparpille (1999) de Jean-Marc Dalpé et Le Coulonneux (1998) de Simone Chaput. Cette image de la maison qui brûle me semble symbolique en effet d'un certain rapport au temps et à l'altérité, qui se manifeste autant sur les berges indécises de la Petitcodiac que sur les rives de la rivière Rouge 
et dans les espaces évanescents du Nord ontarien. Dans tous ces lieux où affleurent péniblement les traces et les reliquats de la présence française et où s'impose de façon implacable le modèle socioculturel nord-américain — qui tend justement à occulter ces traces et ces reliquats — une conscience singulière du temps se fait jour, conscience perceptible dans les romans de Daigle, Poliquin, Dalpé et Chaput. Mais pour bien saisir les tenants et aboutissants de cette image de la maison qui brûle, il importe tout d'abord de faire un détour inattendu dans le temps pour remonter jusqu'à un roman de Lionel Groulx publié en 1932, Au Cap Blomidon, roman qui s'avère emblématique du phénomène qui fait l'objet de cette étude ${ }^{1}$.

\section{Un petit voyage dans le temps}

Dans Au Cap Blomidon, qu'on peut qualifier de roman de politique-fiction, l'abbé Groulx s'est plu à imaginer la reconquête de l'Acadie perdue par un descendant des déportés de 1755 qui, contre toute attente, quitte la province de Québec pour aller s'établir en Nouvelle-Écosse dans le but de reprendre possession de la terre de ses ancêtres. Pour écrire son roman, l'abbé Groulx s'est inspiré d'un voyage qu'il avait fait dans les provinces maritimes en 1915, mais il s'est aussi documenté de façon systématique, consultant de nombreux ouvrages consacrés à la déportation des Acadiens, dont le monumental essai d'Émile Lauvrière, La tragédie d'un peuple, publié à Paris

\footnotetext{
1 Sur la question de la mémoire au Canada français, on pourra consulter les études regroupées dans Anne Gilbert, Michel Bock et Joseph Yvon Thériault (dir.) (2009), Entre lieux et mémoire. L'inscription de la francophonie canadienne dans la durée.
} 
en $1922^{2}$. Dans ce livre, l'historien français insiste sur les affres du Grand Dérangement, en s'indignant du sort fait aux déportés, qui ont vu leurs familles séparées et leurs maisons incendiées. Le premier tome de La tragédie d'un peuple se clôt d'ailleurs sur une vision apocalyptique des villages acadiens livrés à l'élément destructeur :

Sous les yeux de ces survivants, pendant six jours, sévit donc l'incendie; pendant six jours flambèrent une à une toutes les maisons de bois des riants villages acadiens qu'avait fait surgir l'allègre labeur de quatre générations. Quand les vents d'hiver eurent disséminé les lourdes volutes de fumée et les cendres épaisses de ce « colossal » incendie, il ne régna plus dans le noir désert que le silence, il ne resta plus [...] que les puits peut-être empoisonnés, [...] les cheminées noircies, les digues apparemment inutiles, et les saules qui dans la tristesse des lieux semblaient pleurer le deuil d'une nation anéantie. Les derniers Acadiens, qui, parqués sur la rive, purent de leurs yeux épouvantés contempler l'horrible conflagration, durent alors comprendre ce que valaient les promesses anglaises, l'honneur britannique [...] Au pays de leurs ancêtres, il n'y avait plus de foyer pour eux; ils n'avaient plus de patrie. Sans retourner la tête, ils devaient s'embarquer pour l'inconnu, fuir à jamais. ${ }^{3}$ (p. 500-501)

Cette image des maisons qui brûlent détermine, dans $A u$ Cap Blomidon, tout un champ sémantique construit autour du motif des ruines éparses et des traces évanescentes du passé. Dans l'Acadie perdue de Lionel Groulx, l'apocalypse a eu lieu et il ne reste presque plus rien du temps passé. Si on fait

\footnotetext{
2 Consulter à ce sujet l'article de Stéphane Stapinski (1993), «L'intégration d'un document historique à un récit de fiction: l'exemple d'Au cap Blomidon de Lionel Groulx ».

3 Émile Lauvrière, La tragédie d'un peuple, tome 1, Paris, Bossard, 1922, p. 500-501. Il convient de noter au passage que cette image de la maison qui brûle est aussi évoquée avec force dans Les anciens Canadiens (1864) de Philippe Aubert de Gaspé père.
} 
abstraction du discours historiographique et de la tradition orale, dont la nature immatérielle symbolise bien le fait que la mémoire collective est toujours menacée d'anéantissement, l'Acadie perdue ne subsiste que de façon extrêmement fragile et ténue, sous la forme de quelques débris du passé, comme en témoigne bien l'extrait suivant :

- Et ce pauvre chaulage, demande Paul, c'est tout ce qui reste de mes vieux parents?

- Des tiens, des miens, comme de tous les autres. Nos gens une fois capturés et expulsés, les Anglais, la torche à la main, se mettent à brûler et à détruire. Les granges, les maisons, l'église de Saint-Joseph des Canards, tout y passe. [...] Ce qui reste des Acadiens, reprend Jean, qu'une grande tristesse envahit, regarde, tu l'as sous les yeux : quelques caves béantes, de vieux saules, le long des anciennes routes; là-bas, à la Grand'Prée, le vieux puits, la brimbale, quelques pierres de la vieille église et la croix du cimetière. Des ruines, encore des ruines, des souvenirs qui pleurent, puis ces terres, ces vergers, ces grandes digues qui ont fait la fortune des bourreaux. (p. 86-87)

Par une cruelle ironie, cette Acadie des origines est devenue un véritable jardin d'Éden et se trouve décrite comme un «vaste verger ininterrompu» (p. 78) : «Partout les arbres ployaient sous le fardeau des beaux fruits, tassés et inclinés comme des grappes géantes. Un arôme aux mille senteurs flottait dans l'air» (p. 78). Paradoxalement, ce lieu presque intemporel est travaillé par les signes de la modernité, qu'il s'agisse des bâtiments de ferme, de la machinerie agricole et surtout de l'automobile, omniprésente dans le paysage. Dans une étude consacrée au roman québécois des années 1930, Klaus-Dieter Ertler a bien noté cette importance, pour le moins inattendue, de l'automobile dans Au Cap Blomidon :

Avec ce roman de 1932, nous nous trouvons face à la situation paradoxale d'un discours agriculturiste, national et catholique, 
qui intègre sans la moindre résistance l'avalanche discursive propre à la modernité, et ce, avant tout, par le biais de l'automobile. Le texte forme ainsi un conglomérat discursif dont les contradictions dépassent les normes enthymématiques de la société québécoise de l'époque. (p. 536)

Puisant sans distinction dans les rêveries édéniques, les projections utopiques, les visions apocalyptiques et le spectacle de la vie moderne, le roman de Lionel Groulx prend ainsi la forme d'une œuvre composite, qui entretient un rapport original avec les figures du temps. En un sens, $\mathrm{Au} C a p$ Blomidon ne s'avère pas seulement emblématique, mais aussi programmatique des romans qui seront publiés dans son sillage. Certes, loin de moi l'idée de faire du roman de l'abbé Groulx une œuvre qui aurait servi de modèle pour les romanciers issus du Canada francophone. Toujours est-il qu'Au Cap Blomidon me semble mettre en place plusieurs des éléments temporels qui vont bientôt structurer les romans écrits en français à l'extérieur du Québec. Ce roman met ainsi en lumière la nécessité vitale, dans le contexte d'une Amérique française en voie de modernisation, mais de plus en plus fragmentée et engagée sur le chemin de la dissolution, de conjuguer certaines visions, actuelles ou prospectives, avec les signes de l'affleurement du passé et les fragments éparpillés de la mémoire collective. C'est d'ailleurs pourquoi l'action du roman de Lionel Groulx se déroule dans un espace où se côtoient deux types de temporalités, le temps sacralisé des origines et le temps profane de la vie moderne, qui en viennent à se télescoper violemment.

Ce télescopage des temps s'avère d'ailleurs une caractéristique importante de plusieurs romans écrits en français à l'extérieur du Québec. Les romans de France Daigle et 
de Jean Babineau, par exemple, juxtaposent des temporalités qui s'entrechoquent, comme dans Pas pire, de Daigle, où les "temps hypermodernes», pour reprendre l'expression de Gilles Lipovetsky, sont mis en relation étroite et constante avec le temps des origines, comme dans les descriptions qui sont faites de la mise en valeur de la rivière Petitcodiac par la famille Irving. Il en va de même dans Gîte, de Babineau, où l'expression de la modernité la plus radicale côtoie la réflexion qui y est menée sur l'exploration généalogique. C'est ce que Lipovetsky nomme le présent paradoxal, «un présent qui ne cesse d'exhumer et de "redécouvrir" le passé » (p.123). Dans le contexte nord-américain, ce télescopage des temporalités s'exprime toutefois selon une logique particulière, à laquelle sont hautement sensibles les collectivités minoritaires, sur lesquelles s'exercent des pressions qui les incitent souvent à faire table rase du passé, notamment du passé collectif, considéré comme folklorique voire ethnique, et de s'ouvrir du même coup à un futur déréalisé, désinvesti collectivement. Tiraillés entre une mémoire en lambeaux, faite de bribes et de signes fugaces, et la valorisation d'un futur hypostasié, les romans canadiens de langue française touchent ainsi de près à la sensibilité littéraire états-unienne et même américaine au sens large, qui a tendance à s'inscrire pour sa part dans une société "sans mémoire", pour reprendre l'expression de Lucien Sfez (p. 21). Dans La nostalgie des origines, l'historien des religions Mircea Eliade avançait d'ailleurs l'hypothèse que " [l]a découverte et la colonisation du Nouveau Monde ont eu lieu sous le signe de l'eschatologie» (p. 167), en mentionnant notamment l'exemple d'un conte fantastique de Nathaniel Hawthorne, intitulé « Earth's Holocaust » (1844), qui propose 
la vision d'un feu de joie cosmique qui détruit la pompe héraldique des anciennes familles aristocratiques, les robes et les sceptres de la royauté et autres symboles d'une vieille institution, ainsi, pour finir, que la totalité de la littérature et de la philosophie européennes. (Ibid., p. 185)

Cette image de l'holocauste surdétermine ainsi celle de la maison qui brûle, magistralement illustrée dans les œuvres d'Émile Lauvrière et de Lionel Groulx.

\section{Retour vers le futur}

Par un singulier retour des choses, il appartient à la romancière France Daigle d'avoir réactivé, à cinquante ans de distance, l'image forte qui avait tant frappé l'imagination des deux historiens, dans son roman intitulé Histoire de la maison qui brûle, sous-titré Vaguement suivi d'un dernier regard sur la maison qui brûle. En dépit de sa modernité certaine et de sa composition extrêmement savante et recherchée, le roman de France Daigle est hanté à son tour par l'image centrale de la maison qui brûle et habité de toutes parts par le texte d'Émile Lauvrière. De façon pour le moins inusitée, le narrateur du récit premier cherche en effet à envoyer par la poste des provisions à nul autre que l'auteur de La tragédie d'un peuple:

Je me dirigeais tranquillement vers la maison de la poste où prendre les trois formulaires pour envoyer des colis outreAtlantique. Je préparais une boîte de vivres pour Émile Lauvrière qui vivait pauvrement à Paris. (p.18)

La présence de l'historien est ainsi intimement liée à l'histoire de la maison qui brûle. En fait, c'est sa propre histoire de la déportation qui prend valeur de modèle paradigmatique dans l'histoire racontée dans le roman de France Daigle; elle 
forme même quelques-uns des «mantras» qui ponctuent le cours de ce dernier :

La tragédie d'un peuple. Om. (p. 19)

Cette page du manuscrit acadien selon laquelle les martyrs emportèrent comme suprême vision de la patrie les sanglantes lueurs d'un incendie qui dévorait granges, maisons, églises. $0 \mathrm{~m}$. (p. 49)

À l'instar d'Au Cap Blomidon, Histoire de la maison qui brûle met en scène différentes temporalités, qui s'entrechoquent mais aussi qui se superposent, à un point tel qu'elles en viennent presque à se confondre. De façon significative, l'histoire de la maison qui brûle prend place en 1953, une date qui renvoie simultanément à la naissance de France Daigle et à la publication du Degré zéro de l'écriture, de Roland Barthes, date providentielle, si on veut, qui formera par ailleurs le cadre d'un roman subséquent de l'auteure racontant sa venue à l'écriture, roman intitulé 1953. Chronique d'une naissance annoncée. Dans Histoire de la maison qui brûle, on assiste à la répétition générale de cette naissance annoncée au moyen d'un récit emboîté dans le récit premier qui raconte justement une histoire en train de se faire, de venir progressivement à l'existence, tout en se confondant avec celle qui lui sert de modèle. Cette histoire est racontée par le même narrateur qui, en route pour la maison de la poste, s'est arrêté pour observer une femme qui restait assise, sans bouger, sur une petite place. On apprend bientôt que cette femme, avec ses deux enfants, a survécu à l'incendie de sa maison, à l'exemple des Acadiens décrits par Lauvrière, et que, comme eux, elle y a jeté un dernier regard, avant que la demeure ne soit entièrement consumée : «Pour cette femme, la maison brûle toujours, n'a jamais cessé de brûler. Les flammes y sont prises de partout et jamais elle ne 
voit l'image finale de la consumation » (p. 92). Cette histoire (sans fin) de la maison qui brûle nous permet de saisir la relation très intime que le passé, aussi fugace soit-il, entretient avec le présent. Le dernier regard sur la maison qui brûle renvoie bien sûr au dernier regard de Loth sur les villes détruites de Sodome et Gomorrhe, regard frappé d'interdit et pourtant figé à jamais sur un monde en train d'être anéanti par le feu du ciel. À cet égard, la succession des premiers mantras composant le roman de France Daigle s'avère symptomatique d'une conception du temps liée à l'abolition des distances, que ce soit entre le passé et le présent ou entre le sujet et son objet. Voici comment se lisent les cinq premiers mantras :

$\mathrm{Au}$ fond de moi, de l'autre côté de la rue, une femme est assise par terre les jambes croisées, le dos droit, les yeux fermés. Om. (p. 9)

Elle ne bouge pas, n'a pas bougé depuis que je la regarde. $0 \mathrm{~m}$. (p. 11)

Entre elle et moi il n'y a que le temps qui passe. Om. (p. 13)

Entendre au loin qu'une charpente veut céder. Om. (p. 15)

Le temps n'est qu'un symbole. Om. (p. 17)

La contemplation de l'objet, situé simultanément à l'intérieur et à l'extérieur du sujet, mais de façon encore plus singulière de l'autre côté du temps («Entre elle et moi il n’y a que le temps qui passe »), contribue à l'abolition de cet écart tant spatial que temporel (« Le temps n'est qu'un symbole»). En conséquence de quoi, faut-il s'étonner que l'histoire de la maison qui brûle renvoie, une fois de plus, au livre de Lauvrière, dont le titre forme d'ailleurs le sixième mantra du roman : « $L a$ tragédie d'un peuple. Om » (p. 19). 
Dans L'Obomsawin de Daniel Poliquin, la tragédie d'un peuple fait place à la destinée d'une petite ville du nord-ouest ontarien, Sioux Junction, qui est en voie d'être abandonnée par ses derniers habitants. Figuration de l'éphémère, cette ville champignon, fondée au XIX siècle par un Canadien français du nom de Charlemagne Ferron et par un immigrant d'origine ukrainienne ayant troqué son patronyme pour celui de Byron Miles, est littéralement en voie de disparaître, tant d'un point de vue géographique que mémoriel, comme en témoigne l'extrait suivant :

La ville n'existe à peu près plus. Il n'y a qu'à regarder les cartes touristiques de l'Ontario : Sioux Junction n'y apparaît nulle part. Quand la ville a fermé, au gré de la demande du bois et du fer, on a cessé de s'y intéresser. Il faut consulter les relevés topographiques du ministère fédéral des Mines pour se rappeler que la ville a encore un nom. À part ça, rien. Un cœur de pomme oublié. (p. 25)

La meilleure façon de retracer l'histoire de la ville serait de recourir aux toiles peintes autrefois par l'enfant prodigue de la ville, Thomas Christophe Obomsawin, un peintre métis connu dans le monde entier et qui descendait en droite ligne d'un des fondateurs de cette ville, encore qu'il soit impossible de dire lequel. Mais voilà : Thomas Christophe Obomsawin est accusé d'avoir mis le feu à sa maison, qui abritait justement les précieuses toiles, et, par conséquent, une part essentielle de la mémoire de Sioux Junction, mémoire qui est désormais inaccessible: «Mais il ne reste plus rien de la maison. Sauf des cendres et les souvenirs de quelques témoins oculaires » (p. 26). L'image de la maison qui brûle se manifeste ainsi de nouveau, bien que ce soit dans un contexte tout à fait différent. Cette fois, l'auteur de l'incendie serait le protagoniste lui-même, ce qui en soi s'avère révélateur d'un nouveau rapport au passé 
et à la mémoire, plus proche de l'imaginaire américain et de son désir inconscient de faire table rase du passé. Il est dit en effet que Thomas Obomsawin détestait ses tableaux historiques, pour la raison qu'il est impossible de représenter le passé, si ce n'est avec les yeux du présent: «On ne peut jamais peindre l'Histoire pure, c'est toujours soi-même et son temps que l'on représente » (p. 113). C'est pourquoi le peintre aurait mis le feu à ses toiles en les aspergeant d'essence, "surtout la grande murale historique » (p. 144); l'incendie de la maison ne serait que le résultat de cet acte visant symboliquement à l'éradication du passé, du moins tel qu'il est représenté :

Ce sont les tableaux qui ont pris feu les premiers, pas de doute là-dessus. Le reste de la maison, surtout faite de rondins, a brûlé au ras en moins d'une heure. On voyait la lueur de l'incendie à des kilomètres à la ronde. [...] Tout ce qu'on sait de manière certaine à propos du rôle d'Obom, c'est qu'il se tenait devant l'incendie sans rien dire, la face noircie. (p. 144)

Ce regard du peintre sur la maison qui brûle rappelle celui des Acadiens déportés décrits par Émile Lauvrière; mais il évoque encore mieux celui de la femme présente dans le roman de France Daigle, un regard curieusement dégagé du passé et comme affranchi du temps :

Il était difficile de lire l'expression sur le visage de la femme à ce moment-là. C'était une expression qui nous est méconnue, une expression de neutralité qui se rapproche justement du om. (1985, p. 100)

Pour en revenir au roman de Daniel Poliquin, on apprend par ailleurs que c'est le biographe du peintre, surnommé le Déprimé, qui lui a proposé de mettre le feu à la maison, dans le but d'éviter que celle-ci soit transformée en un musée qui lui serait dédié, et surtout pour mettre fin à la formidable imposture entourant la carrière de Thomas Obomsawin, ce 
peintre célèbre de par le monde mais qui n'est même pas l'auteur d'une bonne moitié de ses tableaux. C'est pour mettre un terme à cette imposture que le Déprimé suggère à l'Obomsawin d'incendier sa maison :

Regarde ce qu'on va faire : on y aller toé deux, pis on va mettre le feu dedans. Comme ça, plus de mensonge, pour toi et moi. Après, on pourra vivre honnêtement et faire autre chose. Vienstu? On va effacer ton passé pis le mien en même temps. Y a rien comme un bon feu pour ça. (p. 176)

De façon significative, Thomas Obomsawin va passer les derniers jours de sa vie assis sur un banc d'église, sur le bord de la Wicked Sarah, la rivière qui traverse Sioux Junction et qui fait contrepoids, sur le plan symbolique, à l'image de la maison qui brûle. C'est là que, délesté enfin d'un passé factice mais aussi délivré du futur, il peut renouer avec l'odeur de l'eau, une odeur qui le renvoie à ses propres origines, à sa jeunesse et à ses premières amours. Pour l'Obomsawin, l'odeur de l'eau est l'odeur même de la vie, et le roman suggère qu'elle permet d'accéder au nirvana, un peu comme dans le Siddharta de Herman Hesse, un roman qui se termine lui aussi au bord d'une rivière, ce qui nous ramène au «om » inlassablement répété dans le roman de France Daigle. Le roman de Daniel Poliquin se termine d'ailleurs ainsi : «L'Obomsawin. Mort. Célèbre. Immortel » (p. 185). Obom, pourrait-on ajouter. De l'image de la maison qui brûle à celle de l'eau qui renvoie aux origines, le roman met ainsi en scène la question des rapports avec le temps passé, d'autant plus que le destin qui est réservé à Sioux Junction est d'être englouti sous les eaux, en raison de la construction projetée d'un barrage sur la rivière. La consumation de l'Histoire et l'engloutissement des traces du passé se trouvent ainsi au centre du roman de Daniel Poliquin. 
Précisons par ailleurs que dans le contexte franco-ontarien, l'image de la maison qui brûle se trouve parfois investie de façon positive, ce qui tranche avec le contexte acadien, où la même image figure la catastrophe originelle. Le motif de l'incendie régénérateur traduit en effet un rapport au temps qui s'avère moins chargé de lourdeur historique qu'empreint d'une certaine légèreté, fût-elle insoutenable, et cela même si l'incendie en question devait se propager à l'univers entier, comme il est suggéré par ailleurs dans Un vent se lève qui éparpille, de Jean Marc Dalpé, au moment où un feu de forêt menace une petite ville du nord ontarien. Or, ce feu de forêt aurait été allumé par le protagoniste même du roman, dans un mouvement de révolte contre le destin :

et quand Marcel avait entendu à la radio -- est-ce que c'était hier? -- qu'il y en avait un qui menaçait à moins d'une vingtaine de kilomètres de la petite ville, Marcel avait pensé Qu'y brûle, ostie, Qu'y brûle! Qu'y brûle toute! (p. 11)

Principalement associé à la douleur et à la transgression d'un interdit (l'inceste), complètement privé de résonances collectives, le passé ne mérite au fond que d'être éradiqué, effacé à tout jamais, le monde entier dût-il être consumé dans les flammes. Ce rapport au temps pour le moins particulier nous ramène d'ailleurs à l'image de la fête du bazou dans L'Obomsawin: la fête en question consiste à abandonner sur un lac gelé une vieille voiture et à faire des paris sur la date où elle va s'enfoncer dans les profondeurs du lac. Il en va de même dans le roman de Simone Chaput, $L e$ Coulonneux, un roman consacré à l'errance dans le continent nord-américain et au rapport entretenu avec la mémoire, qui montre à son tour l'incendie d'une petite maison, à Saint- 
Boniface, laquelle se consume sous les yeux réjouis de ses occupants, trois jeunes hommes, laissant voir « une expression sotte, épanouie, sur leur visage, qui levaient leurs bouteilles vers les flammes en rigolant » (p. 184). De tragique qu'elle était, l'histoire de la maison qui brûle devient emblématique d'une société désormais sans mémoire, figurée par ces trois jeunes hommes anonymes dont on ne sait rien, sinon qu'ils passent l'essentiel de leur temps à boire de la bière, jouer au poker et aller à la chasse. Pour tous ces jeunes Canadiens, le monde ancien peut flamber à loisir, dans une sorte de feu de joie qui exprime un nouveau rapport au temps, si rapport il y a encore, tant le "présentisme" mis en lumière par les travaux de François Hartog (2003) semble être devenu une des valeurs dominantes de notre société.

\section{Conclusion}

$\mathrm{Au}$ terme de ce chemin sinueux qui nous a conduits de l'essai d'Émile Lauvrière au roman de Lionel Groulx jusqu'à ceux de France Daigle, Daniel Poliquin, Jean Marc Dalpé et Simone Chaput, il semble bien que nous sommes en présence d'une déperdition mémorielle qui est inquiétante à plusieurs égards, parce qu'elle exprime la rupture progressive des liens unissant la francophonie canadienne avec son passé collectif. L'insistance avec laquelle s'impose l'image de la maison qui brûle dans le roman contemporain suggère que la mémoire collective est actuellement menacée de disparition dans la francophonie canadienne. Ceci étant dit, on peut se demander si le regard sur la maison qui brûle, de figé et médusé qu'il était au départ, ne serait pas devenu plus mobile et délesté du poids 
écrasant du passé, instaurant du même coup un nouveau rapport au Soi et à l'Autre. En d'autres mots, par l'image de la maison qui brûle, les romans de Daigle, Poliquin, Dalpé et Chaput nous enseigneraient-ils une façon d'échapper au traumatisme de l'Histoire mais sans tout oublier du même coup? Chez France Daigle en particulier, même si le rapport vital au temps présent est important, cela n'exclut pas cependant la mémoire d'un passé collectif, mais cette mémoire a cessé d'être envahissante et aliénante pour être considérée avec beaucoup plus de légèreté que dans le discours acadien traditionnel. La même légèreté est présente dans les romans de Daniel Poliquin, Jean Marc Dalpé et Simone Chaput, qui sont en bonne partie délestés du poids de la mémoire, mais sans renier complètement celle-ci pour autant.

Pour prendre l'exacte mesure de ce phénomène, il est sans doute nécessaire de revenir, une fois de plus, à la chanson de Zachary Richard citée en épigraphe de cette étude, chanson qui se termine sur cette strophe : "L'étoile de l'ours déchire la nuit, / L'aurore boréale brûle les cieux. / La solitude et l'oubli, / Dansant seul autour des brandons de mon feu. / Dans le Nord Canadien. » Ces quelques vers, qui montrent que le ciel de la nuit, la solitude et l'oubli sont tout ce qui reste au poète pour exprimer sa condition dans un espace-temps désormais sans borne et sans repère, ne sont certes pas sans évoquer l'extrême fragilité des collectivités francophones et leur disparition éventuelle, magnifiquement illustrées par l'image de la maison qui brûle ; pourtant, la nature pérenne de cette image tend à montrer que la mémoire collective n'est pas disparue pour autant, et même que cette mémoire reste quelque chose de vital qui brûle encore dans le froid de la nuit, dans le nord canadien. 


\section{Bibliographie}

BABINEAU, Jean (1998), Gîte, Moncton, Perce-Neige.

BARThES, Roland (1953), Degré zéro de l'écriture, Paris, Seuil.

CHAPUT, Simone (1998), Le Coulonneux, Saint-Boniface, Éditions du Blé.

DAIGLE, France (1985), Histoire de la maison qui brûle. Vaguement suivi d'un dernier regard sur la maison qui brûle, Moncton, Éditions d'Acadie.

--- (1995), 1953. Chronique d'une naissance annoncée, Moncton, Éditions d'Acadie.

--- (1998), Pas pire, Moncton, Éditions d'Acadie.

DALPÉ, Jean Marc (1999), Un vent se lève qui éparpille, Sudbury, Prise de parole.

EliadE, Mircea (1971), La Nostalgie des origines. Méthodologie et histoire des religions, Paris, Gallimard.

ERTLER, Klaus-Dieter (1994), « Automobile — auto - voiture char: les techniques modernes dans les textes narratifs du Québec des années trente », Voix et Images, vol. 19, no 3 (57), p. 532-544.

GILBERT, Anne, Michel Bock et Joseph Yvon ThÉRIAult (dir.) (2009), Entre lieux et mémoire. L'inscription de la francophonie canadienne dans la durée, Ottawa, Presses de l'Université d'Ottawa, coll. "Amérique française ».

GroulX, Lionel (1932), Au Cap Blomidon, Montréal, Granger. 
HaRtoG, François (2003), Régimes d'historicité. Présentisme et expériences du temps, Paris, Seuil.

HESSE, Hermann (1976 [1950]), Siddhartha, Joseph Delage (trad.), Paris, Grasset.

LAUVRIÈRE, Émile (1922), La Tragédie d'un peuple, tome 1, Paris, Bossard.

LIPOVETSKY, Gilles, avec Sébastien ChASLES (2004), Les Temps hypermodernes, Paris, Grasset.

PoLIQUIN, Daniel (1999 [1987]), L'Obomsawin, nouvelle édition, Montréal, Bibliothèque québécoise.

RichaRD, Zachary (1996), « Dans le Nord Canadien », Album Cap Enragé.

SFEZ, Lucien (1988), Critique de la communication, Paris, Seuil.

STAPINSKI, Stéphane (1993), "L'intégration d'un document historique à un récit de fiction: l'exemple d'Au cap Blomidon de Lionel Groulx», Voix et Images, vol. 19, no 1 (55), p. 54-77.

\section{Résumé}

Cet article aborde la question du rapport à l'altérité dans les romans d'expression française à partir de l'étude d'une image récurrente, celle de la maison qui brûle. Cette image, qui apparaît déjà en filigrane dans $A u$ Cap Blomidon (1932) de Lionel Groulx, joue en effet un rôle central dans des romans comme Histoire de la maison qui brûle (1985) de France Daigle, L'Obomsawin (1987) de Daniel Poliquin, Un vent se lève qui éparpille (1999) de Jean Marc Dalpé et Le Coulonneux (1998) de Simone Chaput. L'analyse de l'image de la maison qui 
brûle permet de dégager comment se manifeste, dans tous ces romans, le rapport particulier qui est entretenu avec le temps, l'histoire et la mémoire, ainsi qu'avec l'altérité angloaméricaine. Ce rapport exprime bien la fragilité extrême des communautés francophones du Canada, que ce soit en Acadie, en Ontario ou au Manitoba.

\begin{abstract}
This paper addresses the question of the relation to otherness in French Canadian novels by examining the recurring image of the burning house. This image, which appears as early as in Lionel Groulx's Au Cap Blomidon (1932), indeed plays a central role in novels like Histoire de la maison qui brûle (1985) by France Daigle, L'Obomsawin (1987) by Daniel Poliquin, Un vent se lève qui éparpille (1999) by Jean Marc Dalpé, and Le Coulonneux (1998) by Simone Chaput. The analysis of the burning house image helps to understand how the particular relation to time, history and memory as well as to the AngloAmerican Other takes form in all these novels. This relation expresses well the extreme fragility of Francophone communities in Canada, whether those in Acadie, in Ontario or in Manitoba.
\end{abstract}

